Article

\title{
The Extremal Graphs of Some Topological Indices with Given Vertex $k$-Partiteness
}

\author{
Fang Gao ${ }^{1}$, Xiaoxin Li $^{1}$, Kai Zhou ${ }^{1}$ (D) and Jia-Bao Liu ${ }^{2, *}$ (D) \\ 1 School of Mathematics and Computer Science, Chizhou University, Chizhou 247000, China; \\ gaofang@czu.edu.cn (F.G.); 1xx@czu.edu.cn (X.L.); zk1984@163.com (K.Z.) \\ 2 School of Mathematics and Physics, Anhui Jianzhu University, Hefei 230601, China \\ * Correspondence: liujiabaoad@163.com
}

Received: 12 October 2018; Accepted: 16 November 2018; Published: 21 November 2018

\begin{abstract}
The vertex $k$-partiteness of graph $G$ is defined as the fewest number of vertices whose deletion from $G$ yields a $k$-partite graph. In this paper, we characterize the extremal value of the reformulated first Zagreb index, the multiplicative-sum Zagreb index, the general Laplacian-energy-like invariant, the general zeroth-order Randić index, and the modified-Wiener index among graphs of order $n$ with vertex $k$-partiteness not more than $m$.
\end{abstract}

Keywords: topological index; vertex $k$-partiteness; extremal graph

\section{Introduction}

All graphs considered in this paper are simple, undirected, and connected. Let $G$ be a graph with vertex set $V(G)=\left\{v_{1}, \cdots, v_{n}\right\}$ and edge set $E(G)=\left\{e_{1}, \cdots, e_{m}\right\}$. The degree of a vertex $u \in V(G)$ is the number of edges incident to $u$, denoted by $d_{G}(u)$. The distance between two vertices $u$ and $v$ is the length of the shortest path connecting $u$ and $v$, denoted by $d_{G}(u, v)$. The complement of $G$, denoted by $\bar{G}$, is the graph with vertex set $V(\bar{G})=V(G)$ and edge set $E(\bar{G})=\{u v: u v \notin E(G)\}$. A subgraph of $G$ induced by $H$, denoted by $\langle H\rangle$, is the subgraph of $G$ that has the vertex set $H$, and for any two vertices $u, v \in V(H)$, they are adjacent in $H$ iff they are adjacent in $G$. The adjacency matrix of $G$ is a square $n \times n$ matrix such that its element $a_{i j}$ is one when there is an edge from vertex $u_{i}$ to vertex $u_{j}$, and zero when there is no edge, denoted by $A(G)$. Let $D(G)=\operatorname{diag}\left(d_{1}, d_{2}, \cdots, d_{n}\right)$ be the diagonal matrix of vertex degrees of $G$. The Laplacian matrix of $G$ is defined as $L(G)=D(G)-A(G)$, and the eigenvalues of $L(G)$ are called Laplacian eigenvalues of $G$, denoted by $\mu_{1}, \cdots, \mu_{n}$ with $\mu_{1} \geq \cdots \geq \mu_{n}$. It is well known that $\mu_{n}=0$, and the multiplicity of zero corresponds to the number of connected components of $G$.

A bipartite graph is a graph whose vertex set can be partitioned into two disjoint sets $U_{1}$ and $U_{2}$, such that each edge has an end vertex in $U_{1}$ and the other one in $U_{2}$. A complete bipartite graph, denoted by $K_{s, t}$, is a bipartite graph with $\left|U_{1}\right|=s$ and $\left|U_{2}\right|=t$, where any two vertices $u \in U_{1}$ and $v \in U_{2}$ are adjacent. If every pair of distinct vertices in $G$ is connected by a unique edge, we call $G$ a complete graph. The complete graph with $n$ vertices is denoted by $K_{n}$. An independent set of $G$ is a set of vertices, no two of which are adjacent. A graph $G$ is called $k$-partite if its vertex-set can be partitioned into $k$ different independent sets $U_{1}, \cdots, U_{k}$. When $k=2$, they are the bipartite graphs, and $k=3$ the tripartite graphs. The vertex $k$-partiteness of graph $G$, denoted by $v_{k}(G)$, is the fewest number of vertices whose deletion from $G$ yields a $k$-partite graph. A complete $k$-partite graph, denoted by $K_{s_{1}, \cdots, s_{k}}$, is a $k$-partite graph with $k$ different independent sets $\left|U_{1}\right|=s_{1}, \cdots,\left|U_{k}\right|=s_{k}$, where there is an edge between every pair of vertices from different independent sets.

A topological index is a numerical value that can be used to characterize some properties of molecule graphs in chemical graph theory. Recently, many researchers have paid much attention to 
studying different topological indices. Dimitrov [1] studied the structural properties of trees with minimal atom-bond connectivity index. Li and Fan [2] obtained the extremal graphs of the Harary index. Xu et al. [3] determined the eccentricity-based topological indices of graphs. Hayat et al. [4] studied the valency-based topological descriptors of chemical networks and their applications. Let $G+u v$ be the graph obtained from $G$ by adding an edge $u v \in E(\bar{G})$. Let $I(G)$ be a graph invariant, if $I(G+u v)>I(G)$ (or $I(G+u v)<I(G)$, respectively) for any edge $u v \in E(\bar{G})$, then we call $I(G)$ a monotonic increasing (or decreasing, respectively) graph invariant with the addition of edges [5]. Let $\mathscr{G}_{n, m, k}$ be the set of graphs with order $n$ and vertex $k$-partiteness $v_{k}(G) \leq m$, where $1 \leq m \leq n-k$. In [5-7], the authors have researched several monotonic topological indices in $\mathscr{G}_{n, m, 2}$, such as the Kirchhoff index, the spectral radius, the signless Laplacian spectral radius, the modified-Wiener index, the connective eccentricity index, and so on. Inspired by these results, we extend the parameter of graph partition from two-partiteness to arbitrary $k$-partiteness. Moreover, we study some monotonic topological indices and characterize the graphs with extremal monotonic topological indices in $\mathscr{G}_{n, m, k}$.

\section{Preliminaries}

The join of two-vertex-disjoint graphs $G_{1}, G_{2}$, denoted by $G=G_{1} \vee G_{2}$, is the graph obtained from the disjoint union $G_{1} \cup G_{2}$ by adding edges between each vertex of $G_{1}$ and each of $G_{2}$. It is to say that $V(G)=V\left(G_{1}\right) \cup V\left(G_{2}\right)$ and $E(G)=E\left(G_{1}\right) \cup E\left(G_{2}\right) \cup\left\{u v: u \in V\left(G_{1}\right), v \in V\left(G_{2}\right)\right\}$.

The join operation can be generalized as follows. Let $F=\left\{G_{1}, \cdots, G_{k}\right\}$ be a set of vertex-disjoint graphs and $H$ be an arbitrary graph with vertex set $V(H)=\{1, \cdots, k\}$. Each vertex $i \in V(H)$ is assigned to the graph $G_{i} \in F$.

The $H$-join of the graphs $G_{1}, \cdots, G_{k}$ is the graph $G=H\left[G_{1}, \cdots, G_{k}\right]$, such that $V(G)=$ $\bigcup_{j=1}^{k} V\left(G_{j}\right)$ and:

$$
E(G)=\bigcup_{j=1}^{k} E\left(G_{j}\right) \bigcup\left(\bigcup_{i j \in E(H)}\left\{u v: u \in V\left(G_{i}\right), v \in V\left(G_{j}\right)\right\}\right) .
$$

If $H=K_{2}$, the $H$-join is the usual join operation of graphs, and the complete $k$-partite graph $K_{s_{1}, \cdots, s_{k}}$ can be seen as the $K_{k}$-join graph $K_{k}\left[O_{s_{1}}, \cdots, O_{s_{k}}\right]$, where $O_{s_{i}}$ is an empty graph of order $s_{i}, 1 \leq i \leq k$.

For $U \subseteq V(G)$, let $G-U$ be the graph obtained from $G$ by deleting the vertices in $U$ and the edges incident with them.

Lemma 1. Let $G$ be an arbitrary graph in $\mathscr{G}_{n, m, k}$ and $I(G)$ be a monotonic increasing graph invariant. Then, there exists $k$ positive integers $s_{1}, \cdots, s_{k}$ satisfying $\sum_{i=1}^{k} s_{i}=n-m$, such that $I(G) \leq I(\widehat{G})$ holds for all graphs $G \in \mathscr{G}_{n, m, k}$, where $\widehat{G}=K_{m} \vee\left(K_{k}\left[O_{s_{1}}, \cdots, O_{s_{k}}\right]\right) \in \mathscr{G}_{n, m, k}$, with equality holds if and only if $G \cong \widehat{G}$.

Proof. Choose $\widehat{G} \in \mathscr{G}_{n, m, k}$ with the maximum value of a monotonic increasing graph invariant such that $I(G) \leq I(\widehat{G})$ for all $G \in \mathscr{G}_{n, m, k}$. Assume that the $k$-partiteness of graph $\widehat{G}$ is $m^{\prime}$, then there exists a vertex set $U$ of graph $\widehat{G}$ with order $m^{\prime}$ such that $\widehat{G}-U$ is a $k$-partite graph with $k$-partition $\left\{U_{1}, \cdots, U_{k}\right\}$. For $1 \leq i \leq k$, let $s_{i}$ be the order of $U_{i}$; hence, $n=\sum_{i=1}^{k} s_{i}+m^{\prime}$.

Firstly, we claim that $\widehat{G}-U=K_{k}\left[O_{s_{1}}, \cdots, O_{s_{k}}\right]$. Otherwise, there exists at least two vertices $u \in U_{s_{i}}$ and $v \in U_{s_{j}}$ for some $i \neq j$, which are not adjacent in $\widehat{G}$. By joining the vertices $u$ and $v$, we get a new graph $\widehat{G}+u v$, obviously, $\widehat{G}+u v \in \mathscr{G}_{n, m, k}$. Then, $I(\widehat{G})<I(\widehat{G}+u v)$, which is a contradiction.

Secondly, we claim that $U$ is the complete graph $K_{m^{\prime}}$. Otherwise, there exists at least two vertices $u, v \in U$, which are not adjacent. By connecting the vertices $u$ and $v$, we arrive at a new graph $\widehat{G}+u v$, obviously, $\widehat{G}+u v \in \mathscr{G}_{n, m, k}$. Then, we have $I(\widehat{G})<I(\widehat{G}+u v)$, a contradiction again. 
Using a similar method, we can get $\widehat{G}=K_{m^{\prime}} \vee\left(K_{k}\left[O_{s_{1}}, \cdots, O_{s_{k}}\right]\right)$.

Finally, we prove that $m^{\prime}=m$. If $m^{\prime} \leq m-1$, then $\sum_{i=1}^{k} s_{i}=n-m^{\prime} \geq n-m+1>n-m \geq k$; thus, $\sum_{i=1}^{k} s_{i}>k$. Without loss of generality, we assume that $s_{1} \geq 2$. By moving a vertex $u \in O_{s_{1}}$ to the set of $U$ and adding edges between $u$ and all the other vertices in $O_{s_{1}}$, we get a new graph $\widetilde{G}=K_{m^{\prime}+1} \vee\left(K_{k}\left[O_{s_{1}-1}, O_{s_{2}}, \cdots, O_{s_{k}}\right]\right)$. It is easy to check that $\widetilde{G} \in \mathscr{G}_{n, m, k}$ has $s_{1}-1$ edges more than the graph $\widehat{G}$. By the definition of the monotonic increasing graph invariant, we get $I(\widehat{G})<I(\widetilde{G})$, which is obviously another contradiction.

Therefore, $\widehat{G}$ is the join of a complete graph with order $m$ and a complete $k$-partite graph with order $n-m$. That is to say $\widehat{G}=K_{m} \vee\left(K_{k}\left[O_{s_{1}}, \cdots, O_{s_{k}}\right]\right)$.

The proof of the lemma is completed.

Lemma 2. Let $G$ be an arbitrary graph in $\mathscr{G}_{n, m, k}$ and $I(G)$ be a monotonic decreasing graph invariant. Then, there exists $k$ positive integers $s_{1}, \cdots, s_{k}$ satisfying $\sum_{i=1}^{k} s_{i}=n-m$, such that $I(G) \geq I(\widehat{G})$ holds for all graphs $G \in \mathscr{G}_{n, m, k}$, where $\widehat{G}=K_{m} \vee\left(K_{k}\left[O_{s_{1}}, \cdots, O_{s_{k}}\right]\right) \in \mathscr{G}_{n, m, k}$, with equality holds if and only if $G \cong \widehat{G}$.

\section{Main Results}

In this section, we will characterize the graphs with an extremal monotonic increasing (or decreasing, respectively) graph invariant in $\mathscr{G}_{n, m, k}$. Assume that $n-m=s k+t$, where $s$ is a positive integer and $t$ is a non-negative integer with $0 \leq t<k$.

\subsection{The Reformulated First Zagreb Index, Multiplicative-Sum Zagreb Index, and k-Partiteness}

The first Zagreb index is used to analyze the structure-dependency of total $\pi$-electron energy on the molecular orbitals, introduced by Gutman and Trinajstć [8]. It is denoted by:

$$
M_{1}(G)=\sum_{u v \in E(G)}\left(d_{G}(u)+d_{G}(v)\right)
$$

which can be also calculated as:

$$
M_{1}(G)=\sum_{u \in V(G)} d_{G}(u)^{2}
$$

Todeschini and Consonni [9] considered the multiplicative version of the first Zagreb index in 2010, defined as:

$$
\Pi_{1}(G)=\prod_{u \in V(G)} d_{G}(u)^{2} .
$$

For an edge $e=u v \in E(G)$, we define the degree of $e$ as $d_{G}(e)=d_{G}(u)+d_{G}(v)-2$. Milličević et al. [10] introduced the reformulated first Zagreb index, defined as:

$$
\widetilde{M}_{1}(G)=\sum_{e \in E(G)} d_{G}(e)^{2}=\sum_{u v \in E(G)}\left(d_{G}(u)+d_{G}(v)-2\right)^{2} .
$$

Eliasi et al. [11] introduced another multiplicative version of the first Zagreb index, which is called the multiplicative-sum Zagreb index and defined as:

$$
\Pi_{1}^{*}(G)=\prod_{u v \in E(G)}\left(d_{G}(u)+d_{G}(v)\right) .
$$

They are widely used in chemistry to study the heat information of heptanes and octanes. For some recent results on the fourth Zagreb indices, one can see [12-17]. 
Lemma 3. Let $G$ be a graph with $u, v \in V(G)$. If $u v \in E(\bar{G})$, then $\widetilde{M}_{1}(G)<\widetilde{M}_{1}(G+u v)$.

Lemma 4. Let $G$ be a graph with $u, v \in V(G)$. If $u v \in E(\bar{G})$, then $\Pi_{1}^{*}(G)<\Pi_{1}^{*}(G+u v)$.

Note that $s_{1}, \cdots, s_{k}$ are $k$ positive integers with $\sum_{i=1}^{k} s_{i}=n-m$.

Theorem 1. Let $\widehat{G}$ be a graph of order $n>2$, and the join of a complete graph with order $m$ and a complete $k$-partite graph with order $n-m$ in $\mathscr{G}_{n, m, k}$, i.e., $\widehat{G}=K_{m} \vee\left(K_{k}\left[O_{s_{1}}, \cdots, O_{s_{k}}\right]\right)$. By moving one vertex from the part of $O_{s_{1}}$ to the part of $O_{s_{2}}$, we get a new graph $\widetilde{G}=K_{m} \vee\left(K_{k}\left[O_{s_{1}-1}, O_{s_{2}+1}, \cdots, O_{s_{k}}\right]\right)$. If $s_{1}-1 \geq s_{2}+1$, then $\widetilde{M}_{1}(\widetilde{G})>\widetilde{M}_{1}(\widehat{G})$.

Proof.By the definition of the reformulated first Zagreb index $\widetilde{M}_{1}(G)$, we can calculate as follows:

$$
\widetilde{M}_{1}(\widehat{G})=\frac{m(m-1)}{2}(2 n-4)^{2}+\sum_{i=1}^{k} m s_{i}\left(2 n-s_{i}-3\right)^{2}+\sum_{1 \leq i<j \leq k} s_{i} s_{j}\left(2 n-s_{i}-s_{j}-2\right)^{2} .
$$

Therefore,

$$
\begin{aligned}
\widetilde{M}_{1}(\widetilde{G})-\widetilde{M}_{1}(\widehat{G}) & =m\left(s_{1}-1\right)\left(2 n-s_{1}-2\right)^{2}+m\left(s_{2}+1\right)\left(2 n-s_{2}-4\right)^{2} \\
& +\left(s_{1}-1\right)\left(s_{2}+1\right)\left(2 n-s_{1}-s_{2}-2\right)^{2}-m s_{1}\left(2 n-s_{1}-3\right)^{2} \\
& -m s_{2}\left(2 n-s_{2}-3\right)^{2}-s_{1} s_{2}\left(2 n-s_{1}-s_{2}-2\right)^{2} \\
& +\sum_{i=3}^{k}\left(s_{1}-1\right) s_{i}\left(2 n-s_{1}-s_{i}-1\right)^{2}+\sum_{i=3}^{k}\left(s_{2}+1\right) s_{i}\left(2 n-s_{2}-s_{i}-3\right)^{2} \\
& -\sum_{i=3}^{k} s_{1} s_{i}\left(2 n-s_{1}-s_{i}-2\right)^{2}-\sum_{i=3}^{k} s_{2} s_{i}\left(2 n-s_{2}-s_{i}-2\right)^{2} \\
& =\left(s_{1}-s_{2}-1\right)\left[(5 n+3 p-12) p+(n+p-2)^{2}\right. \\
& +(7 n+8 m-12) \sum_{i=3}^{k} s_{i}+\left(\sum_{i=3}^{k} s_{i}\right)^{2}+\sum_{i=3}^{k} s_{i}\left(3 \sum_{i=3}^{k} s_{i}-4 s_{i}\right) \\
& =\left(s_{1}-s_{2}-1\right)\left[(n-2)^{2}+(7 n-16) m+4 m^{2}\right. \\
& \left.+(7 n+8 m-12) \sum_{i=3}^{k} s_{i}+4\left(\sum_{i=3}^{k} s_{i}\right)^{2}-4 \sum_{i=3}^{k} s_{i}^{2}\right] \\
& >\left(s_{1}-s_{2}-1\right)\left[(n-2)^{2}+(4 n-8) m+4 m^{2}\right] \\
& =\left(s_{1}-s_{2}-1\right)(n-2+2 m)^{2}>0 . \quad \square
\end{aligned}
$$

Note that we have $n-m=s k+t=(k-t) s+t(s+1)$, where $s$ is a positive integer and $t$ is a non-negative integer with $0 \leq t<k$. For simplicity, we write $K_{m} \vee\left(K_{k}\left[\{k-t\} O_{s},\{s\} O_{s+1}\right]\right)=$ $K_{m} \vee\left(K_{k}[\underbrace{O_{s}, \cdots, O_{s}}_{k-t}, \underbrace{O_{s+1}, \cdots, O_{s+1}}_{t}]\right)$. Then, the extremal value and the corresponding graph of the reformulated first Zagreb index $\widetilde{M}_{1}(G)$ can be shown as follows.

Theorem 2. Let $G$ be an arbitrary graph in $\mathscr{G}_{n, m, k}$. Then:

$$
\begin{aligned}
\widetilde{M}_{1}(G) & \leq \frac{m(m-1)}{2}(2 n-4)^{2}+m(n-m)(6 n-3 s-11) \\
& +2(n-m)(n-m-s)(n-s-1)^{2} \\
& +t(s+1)\left[-6(n-s-1)^{2}+n+2 m(5-2 n+s)+(t-2)(s+1)\right],
\end{aligned}
$$


with the equality holding if and only if $G \cong K_{m} \vee\left(K_{k}\left[\{k-t\} O_{s},\{s\} O_{s+1}\right]\right)$.

Proof. By Lemmas 1, 3, and Theorem 1, the extremal graph having the maximum reformulated first Zagreb index in $\mathscr{G}_{n, m, k}$ is the graph $K_{m} \vee\left(K_{k}\left[\{k-t\} O_{s},\{s\} O_{s+1}\right]\right)$.

Let $\widehat{G}=K_{m} \vee\left(K_{k}\left[\{k-t\} O_{s},\{s\} O_{s+1}\right]\right)$.

Then, we obtain that:

$$
\begin{aligned}
\widetilde{M}_{1}(\widehat{G}) & =\frac{m(m-1)}{2}(2 n-4)^{2}+(k-t) m s(2 n-s-3)^{2} \\
& +t m(s+1)(2 n-s-4)^{2}+\frac{t(t-1)}{2}(s+1)^{2}(2 n-2 s-4)^{2} \\
& +\frac{(k-t)(k-t-1)}{2} s^{2}(2 n-2 s-2)^{2}+t(k-t) s(s+1)(2 n-2 s-3)^{2} \\
& =\frac{m(m-1)}{2}(2 n-4)^{2}+m(n-m)(6 n-3 s-11) \\
& +2(n-m)(n-m-s)(n-s-1)^{2} \\
& +t(s+1)\left[-6(n-s-1)^{2}+n+2 m(5-2 n+s)+(t-2)(s+1)\right] .
\end{aligned}
$$

Theorem 3. Let $\widehat{G}$ be a graph of order $n>2$, and the join of a complete graph with order $m$ and a complete $k$-partite graph with order $n-m$ in $\mathscr{G}_{n, m, k}$, i.e., $\widehat{G}=K_{m} \vee\left(K_{k}\left[O_{s_{1}}, \cdots, O_{s_{k}}\right]\right)$. If $s_{1}-1 \geq s_{2}+1$, by moving one vertex from the part of $O_{s_{1}}$ to the part of $O_{s_{2}}$, we get a new graph $\widetilde{G}=K_{m} \vee\left(K_{k}\left[O_{s_{1}-1}, O_{s_{2}+1}, \cdots, O_{s_{k}}\right]\right)$. Then, $\Pi_{1}^{*}(\widetilde{G})>\Pi_{1}^{*}(\widehat{G})$.

Proof. By the definition of the multiplicative-sum Zagreb index $\Pi_{1}^{*}(G)$, it is easy to see that:

$$
\Pi_{1}^{*}(\widehat{G})=(2 n-2)^{\frac{m(m-1)}{2}} \Pi_{i=1}^{k}\left(2 n-s_{i}-1\right)^{m s_{i}} \prod_{1 \leq i<j \leq k}\left(2 n-s_{i}-s_{j}\right)^{s_{i} s_{j}} .
$$

Hence,

$$
\begin{aligned}
\frac{\Pi_{1}^{*}(\widetilde{G})}{\Pi_{1}^{*}(\widehat{G})} & =\left(2 n-s_{1}-s_{2}\right)^{\left(s_{1}-s_{2}-1\right)} \frac{2 n-s_{2}-2}{2 n-s_{1}-1} a^{m\left(s_{1}-1\right)} b^{m s_{2}} \\
& \Pi_{i=3}^{k} c^{\left(s_{1}-1\right) s_{i}} \prod_{i=3}^{k} d^{s_{2} s_{i}} \Pi_{i=3}^{k}\left(\frac{2 n-s_{2}-s_{i}-1}{2 n-s_{1}-s_{i}}\right)^{s_{i}} \\
> & (a b)^{m s_{2}} \prod_{i=3}^{k}(c d)^{s_{2} s_{i}},
\end{aligned}
$$

where $a=\frac{2 n-s_{1}}{2 n-s_{1}-1}, b=\frac{2 n-s_{2}-2}{2 n-s_{2}-1}, c=\frac{2 n-s_{1}-s_{i}+1}{2 n-s_{1}-s_{i}}, d=\frac{2 n-s_{2}-s_{i}-1}{2 n-s_{2}-s_{i}}$.

By a simple calculation, we have:

$$
\begin{gathered}
\left(2 n-s_{1}\right)\left(2 n-s_{2}-2\right)-\left(2 n-s_{1}-1\right)\left(2 n-s_{2}-1\right)=s_{1}-s_{2}-1>0, \\
\left(2 n-s_{1}-s_{i}+1\right)\left(2 n-s_{2}-s_{i}-1\right)-\left(2 n-s_{1}-s_{i}\right)\left(2 n-s_{2}-s_{i}\right)=s_{1}-s_{2}-1>0 .
\end{gathered}
$$

Therefore, $\frac{\Pi_{1}^{*}(\widetilde{G})}{\Pi_{1}^{*}(\tilde{G})}>1$.

Theorem 4. Let $G$ be an arbitrary graph in $\mathscr{G}_{n, m, k}$. Then:

$$
\begin{aligned}
\Pi_{1}^{*}(G) & \leq(2 n-2)^{\frac{m(m-1)}{2}}(2 n-s-1)^{m s(k-t)}(2 n-s-2)^{m(s+1) t} \\
& (2 n-2 s)^{\frac{s^{2}(k-t)(k-t-1)}{2}}(2 n-2 s-2)^{\frac{(s+1)^{2} t(t-1)}{2}}(2 n-2 s-1)^{s(s+1) t(k-t),}
\end{aligned}
$$

with the equality holding if and only if $G \cong K_{m} \vee\left(K_{k}\left[\{k-t\} O_{s},\{s\} O_{s+1}\right]\right)$. 
Proof. By Lemmas 1, 4, and Theorem 3, the extremal graph having the maximum multiplicative-sum Zagreb index in $\mathscr{G}_{n, m, k}$ should be the graph $K_{m} \vee\left(K_{k}\left[\{k-t\} O_{s},\{s\} O_{s+1}\right]\right)$.

Let $\widehat{G}=K_{m} \vee\left(K_{k}\left[\{k-t\} O_{s},\{s\} O_{s+1}\right]\right)$. We get that,

$$
\begin{aligned}
\Pi_{1}^{*}(\widehat{G}) & =(2 n-2)^{\frac{m(m-1)}{2}}(2 n-s-1)^{m s(k-t)}(2 n-s-2)^{m(s+1) t} \\
& (2 n-2 s)^{\frac{s^{2}(k-t)(k-t-1)}{2}}(2 n-2 s-2)^{\frac{(s+1)^{2} t(t-1)}{2}}(2 n-2 s-1)^{s(s+1) t(k-t) .}
\end{aligned}
$$

\subsection{The General Laplacian-Energy-Like Invariant and k-Partiteness}

The general Laplacian-energy-like invariant (also called the sum of powers of the Laplacian eigenvalues) of a graph $G$ is defined by Zhou [18] as:

$$
S_{\alpha}(G)=\sum_{i=1}^{n-1} \mu_{i}^{\alpha}
$$

where $\alpha$ is an arbitrary real number.

$S_{\alpha}(G)$ is the Laplacian-energy-like invariant [19], and the Laplacian energy [20] when $\alpha=\frac{1}{2}$ and $\alpha=2$, respectively. For $\alpha=-1, n S_{-1}(G)$ is equal to the Kirchhoff index [21], and $\alpha=1, \frac{1}{2} S_{1}(G)$ is equal to the number of edges in $G$. For some recent results on the general Laplacian-energy-like invariant, one can see [22-25].

Lemma 5. [18] Let $G$ be a graph with $u, v \in V(G)$. If $u v \in E(\bar{G})$, then $S_{\alpha}(G)>S_{\alpha}(G+u v)$ for $\alpha<0$, and $S_{\alpha}(G)<S_{\alpha}(G+u v)$ for $\alpha>0$.

Lemma 6. [26] If $\mu_{1} \geq \cdots \geq \mu_{i-1} \geq \mu_{i}=0$ are the Laplacian eigenvalues of graph $G$ and $\mu_{1}^{\prime} \geq \cdots \geq$ $\mu_{j-1}^{\prime} \geq \mu_{j}^{\prime}=0$ are the Laplacian eigenvalues of graph $G^{\prime}$, then the Laplacian eigenvalues of $G \vee G^{\prime}$ are:

$$
i+j, \mu_{1}+j, \mu_{2}+j, \cdots, \mu_{i-1}+j, \mu_{1}^{\prime}+i, \mu_{2}^{\prime}+i, \cdots, \mu_{j-1}^{\prime}+i, 0 .
$$

It is well known that Laplacian eigenvalues of the complete graph $K_{p}$ are $0, p, \ldots, p$, and Laplacian eigenvalues of $O_{p}$ are $0,0, \cdots, 0$. Then, the Laplacian eigenvalues of $K_{s_{1}, s_{2}}=O_{s_{1}} \vee O_{s_{2}}$ are $s_{1}+$ $s_{2}, s_{2}, \cdots, s_{2}, s_{1}, \cdots, s_{1}, 0$, where the multiplicity of $s_{2}$ is $s_{1}-1$ and the multiplicity of $s_{1}$ is $s_{2}-1$. The Laplacian eigenvalues of $K_{s_{1}, s_{2}, s_{3}}=K_{s_{1}, s_{2}} \vee O_{s_{3}}$ are $s_{1}+s_{2}+s_{3}, s_{1}+s_{2}+s_{3}, s_{2}+s_{3}, \cdots, s_{2}+$ $s_{3}, s_{1}+s_{3}, \cdots, s_{1}+s_{3}, 0$, where the multiplicity of $s_{2}+s_{3}$ is $s_{1}-1$ and the multiplicity of $s_{1}+s_{3}$ is $s_{2}-1$.

By induction, we have that the Laplacian eigenvalues of $K_{s_{1}, \cdots, s_{k}}$ are $\sum_{i=1}^{k} s_{i}, \cdots, \sum_{i=1}^{k} s_{i}, \sum_{i=1}^{k} s_{i}-$ $s_{1}, \cdots, \sum_{i=1}^{k} s_{i}-s_{1}, \cdots, \sum_{i=1}^{k} s_{i}-s_{k}, \cdots, \sum_{i=1}^{k} s_{i}-s_{k}, 0$, where the multiplicity of $\sum_{i=1}^{k} s_{i}$ is $k-1$ and the multiplicity of $\sum_{i=1}^{k} s_{i}-s_{j}$ is $s_{j}-1$, for $1 \leq j \leq k$.

From Lemma 6 and the above analysis, we obtain the following lemma.

Lemma 7. Let $\widehat{G}$ be a graph of order $n$, and the join of a complete graph with order $m$ and a complete $k$-partite graph with order $n-m$ i.e., $\widehat{G}=K_{m} \vee\left(K_{k}\left[O_{s_{1}}, \cdots, O_{s_{k}}\right]\right)$. Then, the Laplacian eigenvalues of $\widehat{G}$ are $n, \cdots, n, n-s_{1}, \cdots, n-s_{1}, \cdots, n-s_{k}, \cdots, n-s_{k}, 0$, where the multiplicity of $n$ is $m+k-1$ and the multiplicity of $n-s_{j}$ is $s_{j}-1$, for $1 \leq j \leq k$.

Theorem 5. Let $\widehat{G}$ be a graph of order $n>2$, and the join of a complete graph with order $m$ and a complete $k$-partite graph with order $n-m$ in $\mathscr{G}_{n, m, k}$, i.e., $\widehat{G}=K_{m} \vee\left(K_{k}\left[O_{s_{1}}, \cdots, O_{s_{k}}\right]\right)$. If $s_{1}-1 \geq s_{2}+1$, by moving 
one vertex from the part of $O_{s_{1}}$ to the part of $O_{s_{2}}$, we get a new graph $\widetilde{G}=K_{m} \vee\left(K_{k}\left[O_{s_{1}-1}, O_{s_{2}+1}, \cdots, O_{s_{k}}\right]\right)$. Then, $S_{\alpha}(\widetilde{G})<S_{\alpha}(\widehat{G})$ for $\alpha<0$, and $S_{\alpha}(\widetilde{G})>S_{\alpha}(\widehat{G})$ for $0<\alpha<1$.

Proof. By the definition of the general Laplacian-energy-like invariant $S_{\alpha}(G)$ and Lemma 7, we conclude that:

$$
S_{\alpha}(\widehat{G})=(m+k-1) n^{\alpha}+\sum_{i=1}^{k}\left(s_{i}-1\right)\left(n-s_{i}\right)^{\alpha} .
$$

Therefore:

$$
\begin{aligned}
S_{\alpha}(\widetilde{G})-S_{\alpha}(\widehat{G}) & =\left(s_{1}-2\right)\left(n-s_{1}+1\right)^{\alpha}+s_{2}\left(n-s_{2}-1\right)^{\alpha} \\
& -\left(s_{1}-1\right)\left(n-s_{1}\right)^{\alpha}-\left(s_{2}-1\right)\left(n-s_{2}\right)^{\alpha} \\
& =\left(s_{1}-2\right)\left[\left(n-s_{1}+1\right)^{\alpha}-\left(n-s_{1}\right)^{\alpha}\right] \\
& +\left(s_{2}-1\right)\left[\left(n-s_{2}-1\right)^{\alpha}-\left(n-s_{2}\right)^{\alpha}\right]+\left(n-s_{2}-1\right)^{\alpha}-\left(n-s_{1}\right)^{\alpha} .
\end{aligned}
$$

For $\alpha<0$, we have:

$$
\begin{aligned}
S_{\alpha}(\widetilde{G})-S_{\alpha}(\widehat{G}) & <\left(s_{1}-2\right)\left[\left(n-s_{1}+1\right)^{\alpha}-\left(n-s_{1}\right)^{\alpha}\right]+\left(s_{2}-1\right)\left[\left(n-s_{2}-1\right)^{\alpha}-\left(n-s_{2}\right)^{\alpha}\right] \\
& <\left(s_{1}-2\right)\left[\left(n-s_{1}+1\right)^{\alpha}-\left(n-s_{1}\right)^{\alpha}+\left(n-s_{2}-1\right)^{\alpha}-\left(n-s_{2}\right)^{\alpha}\right] \\
& =\left(s_{1}-2\right)\left[f\left(n-s_{1}\right)-f\left(n-s_{2}-1\right)\right],
\end{aligned}
$$

where $f(x)=(x+1)^{\alpha}-x^{\alpha}, f^{\prime}(x)=\alpha(x+1)^{\alpha-1}-\alpha x^{\alpha-1}>0$.

Then, $f\left(n-s_{1}\right)<f\left(n-s_{2}-1\right)$, and $S_{\alpha}(\widetilde{G})<S_{\alpha}(\widehat{G})$.

For $0<\alpha<1$, we have:

$$
\begin{aligned}
S_{\alpha}(\widetilde{G})-S_{\alpha}(\widehat{G}) & >\left(s_{1}-2\right)\left[\left(n-s_{1}+1\right)^{\alpha}-\left(n-s_{1}\right)^{\alpha}\right]+\left(s_{2}-1\right)\left[\left(n-s_{2}-1\right)^{\alpha}-\left(n-s_{2}\right)^{\alpha}\right] \\
& >\left(s_{2}-1\right)\left[\left(n-s_{1}+1\right)^{\alpha}-\left(n-s_{1}\right)^{\alpha}+\left(n-s_{2}-1\right)^{\alpha}-\left(n-s_{2}\right)^{\alpha}\right] \\
& =\left(s_{2}-1\right)\left[f\left(n-s_{1}\right)-f\left(n-s_{2}-1\right)\right],
\end{aligned}
$$

where $f(x)=(x+1)^{\alpha}-x^{\alpha}, f^{\prime}(x)=\alpha(x+1)^{\alpha-1}-\alpha x^{\alpha-1}<0$.

Then, $f\left(n-s_{1}\right)>f\left(n-s_{2}-1\right)$, and $S_{\alpha}(\widetilde{G})>S_{\alpha}(\widehat{G})$.

Theorem 6. Let $G$ be an arbitrary graph in $\mathscr{G}_{n, m, k}$. Then,

for $\alpha<0, S_{\alpha}(G) \geq(m+k-1) n^{\alpha}+(k-t)(s-1)(n-s)^{\alpha}+t s(n-s-1)^{\alpha}$,

for $0<\alpha<1, S_{\alpha}(G) \leq(m+k-1) n^{\alpha}+(k-t)(s-1)(n-s)^{\alpha}+t s(n-s-1)^{\alpha}$,

with the equality holding if and only if $G \cong K_{m} \vee\left(K_{k}\left[\{k-t\} O_{s},\{s\} O_{s+1}\right]\right)$.

Proof. By Lemmas 1, 2, and Theorem 5, the extremal graph having the extremal value of the general Laplacian-energy-like invariant in $\mathscr{G}_{n, m, k}$ should be the graph $K_{m} \vee\left(K_{k}\left[\{k-t\} O_{s},\{s\} O_{s+1}\right]\right)$.

Let $\widehat{G}=K_{m} \vee\left(K_{k}\left[\{k-t\} O_{s},\{s\} O_{s+1}\right]\right)$, then we can verify that $S_{\alpha}(\widehat{G})=(m+k-1) n^{\alpha}+(k-t)(s-1)(n-s)^{\alpha}+t s(n-s-1)^{\alpha}$.

\subsection{The General Zeroth-Order Randić Index and k-Partiteness}

The general zeroth-order Randić index is introduced by Li [27] as:

$$
{ }^{0} R_{\alpha}(G)=\sum_{u \in V(G)}\left(d_{G}(u)\right)^{\alpha},
$$

where $\alpha$ is a non-zero real number. 
${ }^{0} R_{\alpha}(G)$ is the inverse degree [28], the zeroth-Randić index [29], the first Zagreb index [30], and the forgotten index [31] when $\alpha=-1, \alpha=-\frac{1}{2}, \alpha=2$, and $\alpha=3$, respectively. For some recent results on the general zeroth-order Randić index, one can see [32-34].

Lemma 8. Let $G$ be a graph with $u, v \in V(G)$. If $u v \in E(\bar{G})$, then ${ }^{0} R_{\alpha}(G)>{ }^{0} R_{\alpha}(G+u v)$ for $\alpha<0$, and ${ }^{0} R_{\alpha}(G)<{ }^{0} R_{\alpha}(G+u v)$ for $\alpha>0$.

Theorem 7. Let $\widehat{G}$ be a graph of order $n>2$, and the join of a complete graph with order $m$ and a complete $k$-partite graph with order $n-m$ in $\mathscr{G}_{n, m, k}$, i.e., $\widehat{G}=K_{m} \vee\left(K_{k}\left[O_{s_{1}}, \cdots, O_{s_{k}}\right]\right)$. If $s_{1}-1 \geq s_{2}+1$, by moving one vertex from the part of $O_{s_{1}}$ to the part of $O_{s_{2}}$, we get a new graph $\widetilde{G}=K_{m} \vee\left(K_{k}\left[O_{s_{1}-1}, O_{s_{2}+1}, \cdots, O_{s_{k}}\right]\right)$. Then, ${ }^{0} R_{\alpha}(\widetilde{G})<{ }^{0} R_{\alpha}(\widehat{G})$ for $\alpha<0$, and ${ }^{0} R_{\alpha}(\widetilde{G})>{ }^{0} R_{\alpha}(\widehat{G})$ for $0<\alpha<1$.

Proof. By the definition of the general zeroth-order Randić index ${ }^{0} R_{\alpha}(G)$, we have:

$$
{ }^{0} R_{\alpha}(\widehat{G})=m(n-1)^{\alpha}+\sum_{i=1}^{k} s_{i}\left(n-s_{i}\right)^{\alpha}
$$

Then,

$$
\begin{aligned}
{ }^{0} R_{\alpha}(\widetilde{G})-{ }^{0} R_{\alpha}(\widehat{G}) & =\left(s_{1}-1\right)\left(n-s_{1}+1\right)^{\alpha}-s_{1}\left(n-s_{1}\right)^{\alpha} \\
& +\left(s_{2}+1\right)\left(n-s_{2}-1\right)^{\alpha}-s_{2}\left(n-s_{2}\right)^{\alpha} \\
& =\left(n-s_{2}-1\right)^{\alpha}-\left(n-s_{1}\right)^{\alpha} \\
& +\left(s_{1}-1\right)\left[\left(n-s_{1}+1\right)^{\alpha}-\left(n-s_{1}\right)^{\alpha}\right]+s_{2}\left[\left(n-s_{2}-1\right)^{\alpha}-\left(n-s_{2}\right)^{\alpha}\right] .
\end{aligned}
$$

For $\alpha<0$, we have:

$$
\begin{aligned}
{ }^{0} R_{\alpha}(\widetilde{G})-{ }^{0} R_{\alpha}(\widehat{G}) & <\left(s_{1}-1\right)\left[\left(n-s_{1}+1\right)^{\alpha}-\left(n-s_{1}\right)^{\alpha}+\left(n-s_{2}-1\right)^{\alpha}-\left(n-s_{2}\right)^{\alpha}\right] \\
& =\left(s_{1}-1\right)\left[f\left(n-s_{1}\right)-f\left(n-s_{2}-1\right)\right]
\end{aligned}
$$

where $f(x)=(x+1)^{\alpha}-x^{\alpha}, f^{\prime}(x)=\alpha(x+1)^{\alpha-1}-\alpha x^{\alpha-1}>0$. Then, $f\left(n-s_{1}\right)<f\left(n-s_{2}-1\right)$, ${ }^{0} R_{\alpha}(\widetilde{G})<{ }^{0} R_{\alpha}(\widehat{G})$.

For $0<\alpha<1$, we have:

$$
\begin{aligned}
{ }^{0} R_{\alpha}(\widetilde{G})-{ }^{0} R_{\alpha}(\widehat{G}) & >s_{2}\left[\left(n-s_{1}+1\right)^{\alpha}-\left(n-s_{1}\right)^{\alpha}+\left(n-s_{2}-1\right)^{\alpha}-\left(n-s_{2}\right)^{\alpha}\right] \\
& =s_{2}\left[f\left(n-s_{1}\right)-f\left(n-s_{2}-1\right)\right],
\end{aligned}
$$

where $f(x)=(x+1)^{\alpha}-x^{\alpha}, f^{\prime}(x)=\alpha(x+1)^{\alpha-1}-\alpha x^{\alpha-1}<0$.

Then, $f\left(n-s_{1}\right)>f\left(n-s_{2}-1\right), R_{\alpha}(\widetilde{G})>R_{\alpha}(\widehat{G})$.

Theorem 8. Let $G$ be an arbitrary graph in $\mathscr{G}_{n, m, k}$. Then,

for $\alpha<0,{ }^{0} R_{\alpha}(G) \geq m(n-1)^{\alpha}+(k-t) s(n-s)^{\alpha}+t(s+1)(n-s-1)^{\alpha}$,

for $0<\alpha<1,{ }^{0} R_{\alpha}(G) \leq m(n-1)^{\alpha}+(k-t) s(n-s)^{\alpha}+t(s+1)(n-s-1)^{\alpha}$,

with the equality holding if and only if $G \cong K_{m} \vee\left(K_{k}\left[\{k-t\} O_{s},\{s\} O_{s+1}\right]\right)$.

Proof. By Lemma 8 and Theorem 7, in view of Lemmas 1 and 2, the extremal graph having the extremal value of the general zeroth-order Randić index in $\mathscr{G}_{n, m, k}$ should be the graph $K_{m} \vee\left(K_{k}[\{k-\right.$ t\} $\left.\left.O_{s},\{s\} O_{s+1}\right]\right)$.

Let $\widehat{G}=K_{m} \vee\left(K_{k}\left[\{k-t\} O_{s},\{s\} O_{s+1}\right]\right)$. By a simple calculation, we have ${ }^{0} R_{\alpha}(\widehat{G})=m(n-1)^{\alpha}+(k-t) s(n-s)^{\alpha}+t(s+1)(n-s-1)^{\alpha}$. 


\subsection{The Modified-Wiener Index and k-Partiteness}

The modified-Wiener index is defined by Gutman [35] as:

$$
W_{\lambda}(G)=\sum_{u, v \in V(G)} d_{G}^{\lambda}(u, v),
$$

where $\lambda$ is a non-zero real number.

Lemma 9. Let $G$ be a graph with $u, v \in V(G)$. If $u v \in E(\bar{G})$, then $W_{\lambda}(G)<W_{\lambda}(G+u v)$ for $\lambda<0$, and $W_{\lambda}(G)>W_{\lambda}(G+u v)$ for $\lambda>0$.

Theorem 9. Let $\widehat{G}$ be a graph of order $n>2$, and the join of a complete graph with order $m$ and a complete k-partite graph with order $n-m$ in $\mathscr{G}_{n, m, k}$, i.e., $\widehat{G}=K_{m} \vee\left(K_{k}\left[O_{s_{1}}, \cdots, O_{s_{k}}\right]\right)$. If $s_{1}-1 \geq s_{2}+1$, by moving one vertex from the part of $O_{s_{1}}$ to the part of $O_{s_{2}}$, we get a new graph $\widetilde{G}=K_{m} \vee\left(K_{k}\left[O_{s_{1}-1}, O_{s_{2}+1}, \cdots, O_{s_{k}}\right]\right)$. Then, $W_{\lambda}(\widetilde{G})>W_{\lambda}(\widehat{G})$ for $\lambda<0$, and $W_{\lambda}(\widetilde{G})<W_{\lambda}(\widehat{G})$ for $\lambda>0$.

Proof. By the definition of the modified-Wiener index $W_{\lambda}(G)$, we have the following result.

$$
W_{\lambda}(\widehat{G})=\frac{m(m-1)}{2}+\sum_{i=1}^{k} \frac{s_{i}\left(s_{i}-1\right)}{2} 2^{\lambda}+\sum_{i=1}^{k} m s_{i}+\sum_{1 \leq i<j \leq k} s_{i} s_{j}
$$

Then,

$$
\begin{aligned}
W_{\lambda}(\widetilde{G})-W_{\lambda}(\widehat{G}) & =\frac{\left(s_{1}-1\right)\left(s_{1}-2\right)}{2} 2^{\lambda}+\frac{\left(s_{2}+1\right) s_{2}}{2} 2^{\lambda}+m\left(s_{1}-1\right) \\
& +m\left(s_{2}+1\right)+\left(s_{1}-1\right)\left(s_{2}+1\right)+\sum_{i=3}^{k}\left(s_{1}-1\right) s_{i}+\sum_{i=3}^{k}\left(s_{2}+1\right) s_{i} \\
& -\frac{s_{1}\left(s_{1}-1\right)}{2} 2^{\lambda}-\frac{s_{2}\left(s_{2}-1\right)}{2} 2^{\lambda}-m s_{1}-m s_{2}-s_{1} s_{2}-\sum_{i=3}^{k} s_{1} s_{i}-\sum_{i=3}^{k} s_{2} s_{i} \\
& =\left(s_{1}-s_{2}-1\right)\left(1-2^{\lambda}\right) .
\end{aligned}
$$

For $\lambda>0$, we have $W_{\lambda}(\widetilde{G})<W_{\lambda}(\widehat{G})$. For $\lambda<0$, we have $W_{\lambda}(\widetilde{G})>W_{\lambda}(\widehat{G})$.

Theorem 10. Let $G$ be an arbitrary graph in $\mathscr{G}_{n, m, k}$. Then,

for $\alpha<0, W_{\lambda}(G) \leq \frac{1}{2}\left[m(m-1)+(n-m)(n+m-s)-(s+1) t+s(n-m+t-k) 2^{\lambda}\right]$, for $\alpha>0, W_{\lambda}(G) \geq \frac{1}{2}\left[m(m-1)+(n-m)(n+m-s)-(s+1) t+s(n-m+t-k) 2^{\lambda}\right]$, with the equality holding if and only if $G \cong K_{m} \vee\left(K_{k}\left[\{k-t\} O_{s},\{s\} O_{s+1}\right]\right)$.

Proof. By Lemma 9 and Theorem 9, in view of Lemmas 1 and 2, the extremal graph having the extremal value of the modified-Wiener index in $\mathscr{G}_{n, m, k}$ should be the graph $K_{m} \vee\left(K_{k}\left[\{k-t\} O_{s},\{s\} O_{s+1}\right]\right)$.

Let $\widehat{G}=K_{m} \vee\left(K_{k}\left[\{k-t\} O_{s},\{s\} O_{s+1}\right]\right)$. Consequently, we have that:

$$
\begin{aligned}
W_{\lambda}(\widehat{G}) & =\frac{m(m-1)}{2}+(k-t) \frac{s(s-1)}{2} 2^{\lambda}+t \frac{s(s+1)}{2} 2^{\lambda}+t m(s+1)+(k-t) m s \\
& =\frac{1}{2}\left[m(m-1)+(n-m)(n+m-s)-(s+1) t+s(n-m+t-k) 2^{\lambda}\right] .
\end{aligned}
$$

\section{Conclusions}

In this paper, we consider connected graphs of order $n$ with vertex $k$-partiteness not more than $m$ and characterize some extremal monotonic graph invariants such as the reformulated first Zagreb index, the multiplicative-sum Zagreb index, the general Laplacian-energy-like invariant, the general 
zeroth-order Randić index, and the modified-Wiener index among these graphs, and we investigate the corresponding extremal graphs of these invariants.

Author Contributions: The authors made equal contributions in the article. All authors read and approved the final manuscript.

Funding: This research was funded by the National Science Foundation of China under Grant 11601006; the China Postdoctoral Science Foundation under Grant 2017M621579; the Postdoctoral Science Foundation of Jiangsu Province under Grant 1701081B; Anhui Provincial Natural Science Foundation under Grant 1708085QA13; the Natural Science Foundation of the Anhui Provincial Education Department under Grant KJ2016A517; and the Project of Chizhou University under Grants 2017ZRZ009, 2016XJXTD02.

Acknowledgments: The authors are grateful to the anonymous reviewers and the editor for the valuable comments and suggestions.

Conflicts of Interest: The authors declare no conflict of interest.

\section{References}

1. Dimitrov, D. On structural properties of trees with minimal atom-bond connectivity index. Discret. Appl. Math. 2014, 172, 28-44. [CrossRef]

2. Li, X.; Fan, Y. The connectivity and the Harary index of a graph. Discret. Appl. Math. 2015, 181, 167-173. [CrossRef]

3. Xu, K.; Alizadeh, Y.; Das, K.C. On two eccentricity-based topological indices of graphs. Discret. Appl. Math. 2017, 233, 240-251. [CrossRef]

4. Hayat, S.; Wang, S.; Liu, J. Valency-based topological descriptors of chemical networks and their applications. Appl. Math. Model. 2018, 60, 164-178. [CrossRef]

5. Chen, H.; Wu, R.; Deng, H. The extremal values of some monotonic topological indices in graphs with given vertex bipartiteness. MATCH Commun. Math. Comput. Chem. 2017, 78, 103-120.

6. Liu, J.; Pan, X. Minimizing Kirchhoff index among graphs with a given vertex bipartiteness. Appl. Math. Comput. 2016, 291, 84-88. [CrossRef]

7. Robbiano, M.; Morales, K.T.; Martin, B.S. Extremal graphs with bounded vertex bipartiteness number. Linear Algebra Appl. 2016, 493, 28-36. [CrossRef]

8. Gutman, I.; Trinajstić, N. Graph theory and molecular orbitals.Total $\pi$-electron energy of alternant hydrocarbons. Chem. Phys. Lett. 1972, 17, 535-538. [CrossRef]

9. Todeschini, R.; Consonni, V. New local vertex invariants and molecular descriptors based on functions of the vertex degrees. Match Commun. Math. Comput. Chem. 2010, 64, 359-372.

10. Milićević, A.; Nikolić, S.; Trinajstić, N. On reformulated Zagreb indices. Mol. Divers. 2004, 8, $393-399$. [CrossRef] [PubMed]

11. Eliasi, M.; Iranmanesh, A.; Gutman, I. Multiplicative versions of first Zagreb index. Match Commun. Math. Comput. Chem. 2012, 68, 217-230.

12. Borovićanin, B.; Furtula, B. On extremal Zagreb indices of trees with given domination number. Appl. Math. Comput. 2016, 279, 208-218. [CrossRef]

13. Gao, W.; Jamil, M.K.; Farahani, M.R. The hyper-Zagreb index and some graph operations. J. Math. Anal. Appl. 2017, 54, 263-275. [CrossRef]

14. Gutman, I.; Furtula, B.; Vukićević, K.; Popivoda, G. On Zagreb indices and coindices. Match Commun. Math. Comput. Chem. 2015, 74, 5-16.

15. Milovanović, E.I.; Milovanović, I.; Dolićanin, E.; Glogić, E. A note on the first reformulated Zagreb index. Appl. Math. Comput. 2016, 273, 16-20. [CrossRef]

16. Wang, C.; Liu, J.; Wang, S. Sharp upper bounds for multiplicative Zagreb indices of bipartite graphs with given diameter. Discret. Appl. Math. 2017, 227, 156-165. [CrossRef]

17. Wang, S.; Wang, C.; Liu, J. On extremal multiplicative Zagreb indices of trees with given domination number. Appl. Math. Comput. 2018, 332, 338-350. [CrossRef]

18. Zhou, B. On sum of powers of the Laplacian eigenvalues of graphs. Linear Algebra Appl. 2008, 429, $2239-2246$. [CrossRef]

19. Liu, J.; Liu, B. A Laplacian-energy-like invariant of a graph. Match Commun. Math. Comput. Chem. 2011, 66, 713-730. 
20. Lazić, M. On the Laplacian energy of a graph. Czechoslovak Math. J. 2006, 56, 1207-1213. [CrossRef]

21. Klein, D.J.; Randić, M. Resistance distance. J. Math. Chem. 1993, 12, 81-95. [CrossRef]

22. Das, K.C.; Xu, K.; Liu, M. On sum of powers of the Laplacian eigenvalues of graphs. Linear Algebra Appl. 2013, 439, 3561-3575. [CrossRef]

23. Liu, M.; Liu, B. A note on sum of powers of the Laplacian eigenvalues of graphs. Appl. Math. Lett. 2011, 24, 249-252. [CrossRef]

24. Qiao, L.; Zhang, S.; Jing, B.; Li, J. Coulson-type integral formulas for the general Laplacian-energy-like invariant of graphs I. J. Math. Anal. Appl. 2016, 435, 1246-1261. [CrossRef]

25. Tian, G.; Huang, T.; Zhou, B. A note on sum of powers of the Laplacian eigenvalues of bipartite graphs. Linear Algebra Appl. 2009, 430, 2503-2510. [CrossRef]

26. Merris, R. Laplacian graph eigenvectors. Linear Algebra Appl. 1998, 278, 221-236. [CrossRef]

27. Li, X.; Zheng, J. A unified approach to the extremal trees for different indices. Match Commun. Math. Comput. Chem. 2005, 54, 195-208.

28. Fajtlowicz, S. On conjectures of graffiti II. Congr. Numer. 1987, 60, 189-197.

29. Klein, L.B.; Hall, L.H. The meaning of molecular connectivity: A bimolecular accessibility model. Croat. Chem. Acta 2002, 75, 371-382.

30. Nikolić, S.; Kovačenić, G.; Milićević, A.; Trinajstić, N. The Zagreb indices 30 years after. Croat. Chem. Acta 2003, 76, 113-124.

31. Furtula, B.; Gutman, I. On forgotten topological index. J. Math. Chem. 2015, 53, 1184-1190. [CrossRef]

32. Chen, Z.; Su, G.; Volkmann, L. Sufficient conditions on the zeroth-order general Randić index for maximally edge-connected graphs. Discret. Appl. Math. 2017, 218, 64-70. [CrossRef]

33. Su, G.; Tu, J.; Das, K.C. Graphs with fixed number of pendent vertices and minimal Zeroth-order general Randić index. Appl. Math. Comput. 2015, 270, 705-710. [CrossRef]

34. Zhang, B.; Zhou, B. On zeroth-order general Randić indices of trees and unicyclic graphs. Match Commun. Math. Comput. Chem. 2007, 58, 139-146.

35. Gutman, I. A property of the Wiener number and its modifications. Indian J. Chem. 1997, 36, 128-132.

(C) 2018 by the authors. Licensee MDPI, Basel, Switzerland. This article is an open access article distributed under the terms and conditions of the Creative Commons Attribution (CC BY) license (http:/ / creativecommons.org/licenses/by/4.0/). 